\title{
Piezospintronic effect in honeycomb antiferromagnets
}

\author{
Camilo Ulloa, ${ }^{1,4, *}$ Roberto E. Troncoso,${ }^{2,3}$ Scott A. Bender, ${ }^{4}$ R. A. Duine, ${ }^{4,5}$ and A. S. Nunez ${ }^{1}$ \\ ${ }^{1}$ Departamento de Física, Facultad de Ciencias Físicas y Matemáticas, Universidad de Chile, Casilla 487-3, Santiago, Chile \\ ${ }^{2}$ Department of Physics, Norwegian University of Science and Technology, NO-7491 Trondheim, Norway \\ ${ }^{3}$ Departamento de Física, Universidad Técnica Federico Santa María, Avenida España 1680, Valparaíso, Chile \\ ${ }^{4}$ Institute for Theoretical Physics, Utrecht University, Princetonplein 5, 3584 CC Utrecht, The Netherlands \\ ${ }^{5}$ Department of Applied Physics, Eindhoven University of Technology, P.O. Box 513, 5600 MB Eindhoven, The Netherlands
}

(Received 10 August 2017; published 14 September 2017)

\begin{abstract}
The emission of pure spin currents by mechanical deformations, the piezospintronic effect, in antiferromagnets is studied. We characterize the piezospintronic effect in an antiferromagnetic honeycomb monolayer in response to external strains. It is shown that the strain tensor components can be evaluated in terms of the spin Berry phase. In addition, we propose an experimental setup to detect the piezospin current generated in the piezospintronic material through the inverse spin Hall effect. Our results apply to a wide family of two-dimensional antiferromagnetic materials without inversion symmetry, such as the transition-metal chalcogenophosphate materials $M \mathrm{P} X_{3}(M=\mathrm{V}, \mathrm{Mn} ; X=\mathrm{S}, \mathrm{Se}, \mathrm{Te})$ and $\mathrm{NiPSe}_{3}$.
\end{abstract}

DOI: 10.1103/PhysRevB.96.104419

\section{INTRODUCTION}

Spintronics is one of the most promising areas in condensed matter from the point of view of development of novel devices that can enhance or directly replace conventional electronics $[1,2]$. This has motivated intense studies to understand the mutual relation between spin currents and magnetic properties [3]. In this context, antiferromagnets (AFs) have recently gained attention due to their favorable properties [4] and abundance in nature [5]. Compared with conventional ferromagnets, AFs lack macroscopic magnetization [7] and, furthermore, can be operative at much higher frequencies than ferromagnets [6]. The absence of stray fields makes them robust against perturbation due to magnetic fields. Moreover, AFs have also opened a new branch in spintronics by hosting topological matter, such as Weyl semimetals [8] and topological insulators [9].

AFs can also be the cornerstone of spin-current generation. It has been shown that spin angular momentum can be transported through $\mathrm{AF} \mid \mathrm{NM}$ (normal metal) heterostructures, in the form of pumped spin and staggered spin currents [10], associated with the dynamics of the magnetization and staggered field (Néel order), respectively. An alternative route for the generation of spin currents has recently been proposed, which is based on the coupling between mechanical distortions and spin degrees of freedom, namely, the piezospintronic effect [11]. Unlike the related piezoelectric [12] and piezomagnetic [13] effects, this phenomenon is restricted to appearing in systems with the concomitance of time reversal $(\mathcal{T})$ and inversion $(\mathcal{I})$ symmetry breaking. Although, in principle, a crystal might display simultaneously the piezoelectric, piezomagnetic, and piezospintronic effects.

From a phenomenological point of view, a magnetic crystal under mechanical deformations gives rise in a linear response to a spin dipolar moment, $P_{\sigma ; j}^{s}=\sum_{k l} \lambda_{\sigma ; j k l} u_{k l}$, with $\lambda$ the piezospintronic pseudotensor [15], where $\sigma$ and $j$ label the spin and position components, respectively, and $u_{k l}=$ $\left(\partial_{l} u_{k}+\partial_{k} u_{l}\right) / 2$ the strain tensor [16], with $\vec{u}$ the deformation

*C.Ulloa@uu.nl field (sketched in Fig. 1). Under inversion $\lambda$ changes sign and therefore, like the piezoelectric [12] and piezomagnetic [13] effects, the piezospintronic effect is restricted to crystals lacking a center of inversion. Similarly, the spin dipole moment is odd under time reversal, so a system with a nonvanishing piezospintronic tensor must have broken time reversal invariance.

The spin dipolar moment and spin currents are linked [11], through the standard definition [14] of spin currents, by

$$
J_{\sigma, j}^{s}=\frac{\mathrm{d} P_{\sigma ; j}^{s}}{\mathrm{~d} t} .
$$

It is intuitive to realize that crystal classes invariant under $\mathcal{T} \mathcal{I}$ will respond with a pure spin current to an external strain, i.e., displaying exclusively the piezospintronic effect, without giving rise to charge currents. The simple way to understand this is to require $\mathcal{T}$ and $\mathcal{I}$ symmetry breaking, and thus, each spin component manifests opposite piezoelectric effects $[15,16]$. Under inversion the direction of each piezoelectric effect is reversed, while the spin labels remain unchanged and thus there is a reversal of the piezospin current. An additional spin reversal, through the action of $\mathcal{T}$, will restore the original current. Therefore, it is expected that crystal classes invariant simultaneously under spin reversal and spatial inversion will respond with a pure spin current to an external deformation. Some AF structures, like antiferromagnetic honeycombs, represent natural systems in which to explore this effect since they bring together alternating spin configurations that additionally break inversion symmetry.

In this work we present detailed calculations concerning the piezospintronic tensor of an antiferromagnetic honeycomb lattice. These calculations were performed within the tightbinding approximation. In heterostructures such as $\mathrm{AF} \mid \mathrm{NM}$ (normal metal) this effect can be tested via inverse spin Hall effect (ISHE) measurements [17].

The paper is organized as follows. In Sec. II, we compute the piezospintronic properties of an AF honeycomb lattice. In Sec. III we propose an experimental setup to measure the piezospintronic spin current generated at the interface with an 


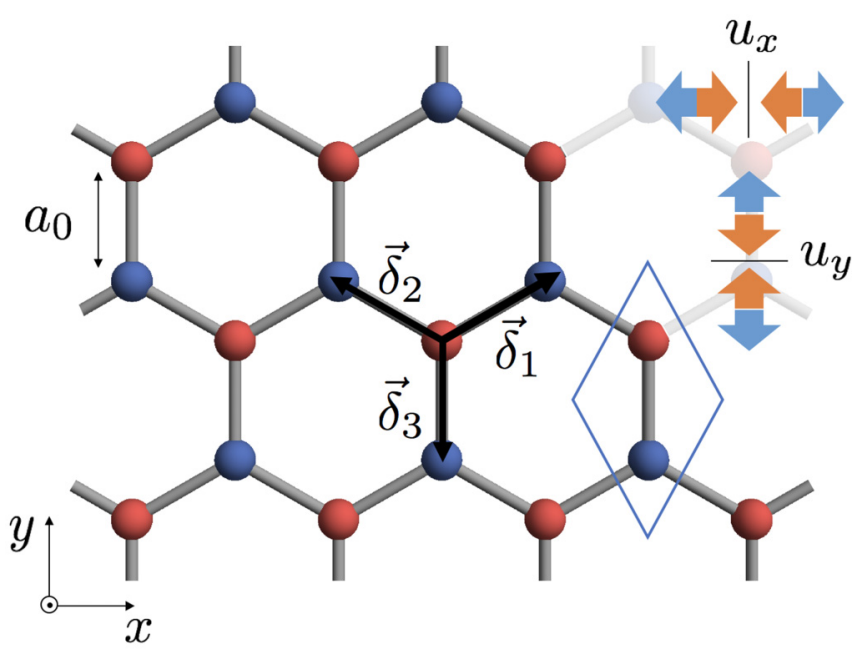

FIG. 1. Antiferromagnetic honeycomb lattice in the $x y$ plane. Red and blue spheres represent the different spin species, spin-up and -down, respectively, whose spin polarizations are along the $z$ direction. Note that this structure lacks $\mathcal{T}$ and $\mathcal{I}$ symmetries but is invariant under the $\mathcal{T} \mathcal{I}$ transformation, thus fulfilling the requirements to exhibit a pure piezospintronic response. In the tight-binding approximation the first nearest neighbors of each site are described by the vectors $\vec{\delta}_{i}$, with $a_{0}$ the unperturbed lattice constant. Strains along the $x$ and $y$ directions are schematically depicted and represented by strain deformation fields $u_{x}$ and $u_{y}$, respectively.

NM through the ISHE in the NM. Finally, we finish in Sec. IV with discussion and conclusions.

\section{ANTIFERROMAGNETIC HONEYCOMB}

\section{A. Model}

We consider a honeycomb lattice with a staggered spin array lying in the $x y$ plane, as described in Fig. 1. The antiparallel lattices of spins, represented by the red and blue spheres in Fig. 1, are oriented along the $z$ axis with spin polarization $\Omega_{i}^{\mathrm{A}, \mathrm{B}}= \pm \Delta \mathbf{z}$. Under spatial inversion around the center of the unit cell, represented by the rhombus in Fig. 1, the position of both sublattices is reversed, i.e., the blue and red spheres are interchanged. A subsequent time reversal operation flips the local spin and thus reverts the effect of spatial inversion. The system is invariant under $\mathcal{T} \mathcal{I}$ and therefore we expect it to display a pure piezospintronic effect. Complementing the local exchange term in our model we also consider hopping to nearest neighbors. The net Hamiltonian is

$$
\mathcal{H}=-\sum_{\langle\boldsymbol{i}, \boldsymbol{j}\rangle, \sigma} \mathrm{t}_{\boldsymbol{i} \boldsymbol{j}}\left(c_{\boldsymbol{i} \sigma}^{\dagger} c_{\boldsymbol{j} \sigma}+\text { H.c. }\right)+\Delta \sum_{\boldsymbol{i} ; \sigma, \sigma^{\prime}} \eta_{\boldsymbol{i}} c_{\boldsymbol{i} \sigma}^{\dagger} \sigma_{\sigma \sigma^{\prime}}^{z} c_{\boldsymbol{i} \sigma^{\prime}}
$$

where $c_{i \sigma}^{\dagger}\left(c_{i \sigma}\right)$ is the operator that creates (annihilates) an electron with spin $\sigma$ at site $i$, and $\eta_{i}= \pm 1$, depending on the sublattice. The hopping matrix element is $t_{i j}$, the energy difference between the spin species is $\Delta$, and $\sigma^{z}$ is the $z$ component of the Pauli matrix vector. The Hamiltonian in
Eq. (2) is Fourier transformed to momentum space and written as

$$
\begin{aligned}
\mathcal{H}_{a} & =\left(\begin{array}{cc}
\Delta & \gamma_{k} \\
\gamma_{k}^{*} & -\Delta
\end{array}\right), \quad \mathcal{H}_{b}=\left(\begin{array}{cc}
-\Delta & \gamma_{k} \\
\gamma_{k}^{*} & \Delta
\end{array}\right), \\
\mathcal{H} & =\left(\begin{array}{cc}
\mathcal{H}_{a} & 0 \\
0 & \mathcal{H}_{b}
\end{array}\right),
\end{aligned}
$$

with $\gamma_{k}=\sum_{i} \mathrm{t}_{i, i+\vec{\delta}_{i}} \exp \left(i \vec{k} \cdot \vec{\delta}_{i}\right)$. In the following we label $\mathrm{t}_{j}$ the hopping amplitude connecting a site with its neighbor $\vec{\delta}_{j}$ (see Fig. 1). At this point we can draw an analogy between the model Hamiltonian we are proposing and the tight-binding model of boron-nitride (BN) monolayers [18]. Sharing the honeycomb structure we see how our model reduces to the BN for each spin species, but with an opposite role for each sublattice. The effect we are looking for follows from the piezoelectric response of $\mathrm{BN}$ and will share all symmetry properties with it.

\section{B. Piezospintronic tensor of a honeycomb antiferromagnet}

As the crystal belongs to the point group $\overline{6} m 2$ (D3h) [15], following the symmetry analysis of $\mathrm{BN}$ we can conclude the following property of the piezospintronic tensor [18]: all the components of the tensor are 0 except for

$$
\lambda_{z ; y y y}=-\lambda_{z ; y x x}=-\lambda_{z ; x y x} .
$$

Our task is then reduced to the evaluation of only one of the components of the tensor, e.g., $\lambda_{z ; y y y}$. We evaluate the net spin dipolar moment created by a deformation of the lattice along the $y$ direction. With the deformation the different hopping amplitudes will change; a simple geometrical analysis leads to

$$
d \mathrm{t}_{1}=d \mathrm{t}_{2}=\frac{1}{2} d \mathrm{t}_{3}=\left(\frac{\partial \mathrm{t}}{\partial a}\right) d u_{y y},
$$

where $\mathrm{t}$ is the hopping amplitude at an interatomic distance $a$. The net spin dipolar moment generated is given by [19]

$$
\mathrm{d} \mathbf{P}_{z ; y}^{S}=\mathbf{A}_{z, y}^{\mathrm{t}_{1}} \mathrm{dt}_{1}+\mathbf{A}_{z, y}^{\mathrm{t}_{2}} \mathrm{dt}_{2}+\mathbf{A}_{z, y}^{\mathrm{t}_{3}} \mathrm{dt}_{3},
$$

where $\mathbf{A}_{z, y}^{\mathrm{t}_{\alpha}}$ is defined as

$$
\mathbf{A}_{z, y}^{\mathbf{t}_{\alpha}} \equiv \frac{\partial \mathbf{P}_{z ; y}^{s}}{\partial \mathbf{t}_{\alpha}}
$$

and can be evaluated in terms of spin Berry phases [11,20], which depend on the electronic Bloch states $\left|\phi_{\nu}\right\rangle$ as

$$
\mathbf{A}_{i, j}^{\mathrm{t}_{\alpha}}=-\sum_{v} \int_{\mathrm{BZ}} \frac{\mathrm{d}^{2} k}{(2 \pi)^{2}} n_{v}(k) \operatorname{Im}\left\langle\frac{\partial \phi_{v}}{\partial k_{j}}\left|\sigma_{i}\right| \frac{\partial \phi_{v}}{\partial \mathrm{t}_{\alpha}}\right\rangle .
$$

The symmetry of the hexagonal lattice enforces a relation among the different $\mathbf{A}_{i, j}^{\mathrm{t}_{\alpha}}$ that reads $\mathbf{A}_{z, y}^{\mathrm{t}_{1}}=\mathbf{A}_{z, y}^{\mathrm{t}_{2}}=-\frac{1}{2} \mathbf{A}_{z, y}^{\mathrm{t}_{3}}$, leading to the final expression for the piezospintronic tensor,

$$
\lambda_{z ; y y y}=-\frac{1}{2}\left(\frac{\partial \mathrm{t}}{\partial a}\right) \mathbf{A}_{z, y}^{\mathrm{t}_{3}} .
$$

The integrand in the expression for $\mathbf{A}_{z, y}^{t_{3}}$ is displayed in Fig. 2. It displays well-defined maxima around the corners of the Brillouin zone (BZ).

The integral is complicated and needs to be evaluated numerically; the obtained result is displayed in Fig. 3. Around 


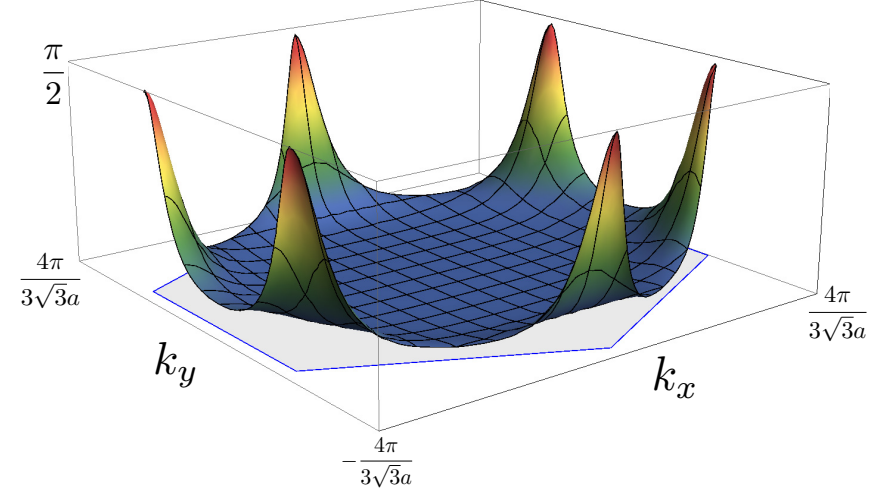

FIG. 2. Berry curvature in the first Brillouin zone of an antiferromagnetic honeycomb [see Eq. (3)]. The integral of this function leads us directly to the value of the piezospintronic tensor $\lambda_{z ; y y y}$ [see Eq. (4)]. Due to the presence of the local energy $\Delta$ there is an asymmetry in the two sublattices, which opens a gap in the spectra around the Dirac points.

one of the Dirac points of the Brillouin zone, however, the Berry curvature can be approximated analytically, yielding

$$
\mathbf{A}_{z, y}^{\mathrm{t}_{3}}=-\frac{\operatorname{sign}(\Delta)}{6 \pi a_{0} \mathrm{t}} .
$$

The independence of the magnitude of $\Delta$ in this result arises from the expression of the eigenstates $\left|\phi_{\nu}\right\rangle$ in the long-wavelength limit. As its $j$ th component is proportional to $k_{j}$ we can replace $\partial_{\mathrm{t}_{j}} \rightarrow \partial_{k_{j}}$, obtaining an expression proportional to a Chern number [21].

\section{DETECTION OF PIEZOSPIN CURRENTS}

Now we propose an experimental setup to perform indirect detection of the spin current generated by the piezospintronic effect. We consider two adjacent materials, as shown in Fig. 4, one being piezospintronic and the other being a normal metal

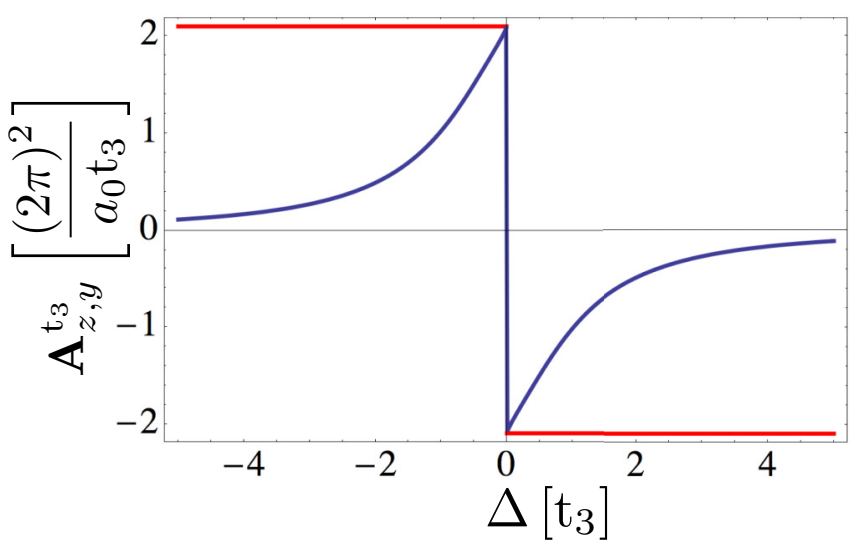

FIG. 3. Result of the integration of $\mathbf{A}_{z, y}^{t_{3}}$ for an antiferromagnetic honeycomb as a function of the local energy $\Delta$. The blue line corresponds to the numerical integration of the Berry curvature in the first Brillouin zone. The red line shows the exact calculation in the long-wavelength limit. The maximum (minimum) value of the curve is $2 \pi / 3(-2 \pi / 3)$ as we expect from the long-wavelength approximation [see Eq. (5)].

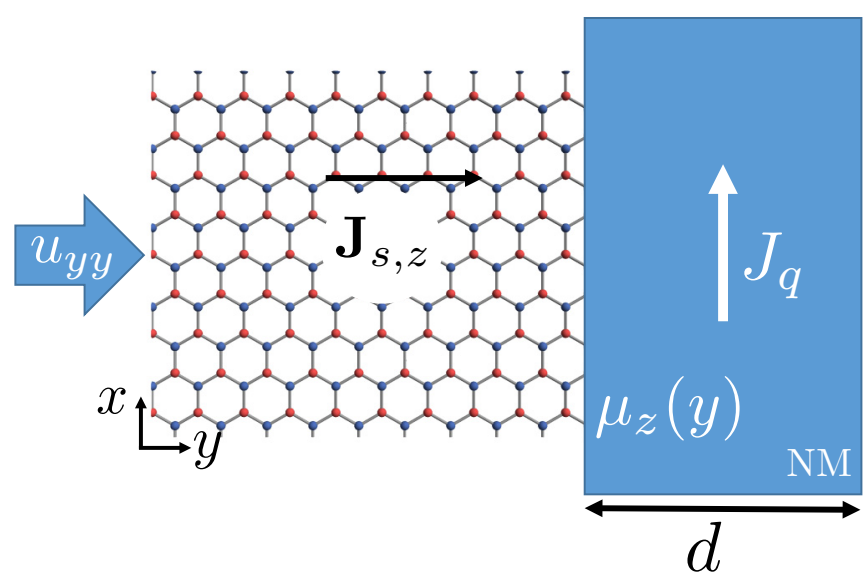

FIG. 4. Schematic setup for the detection of piezospin currents. The proposal is based on similar geometries as used for measurement of the spin Seebeck effect [25]. Under a strain $u_{y y}$ a spin current $\mathbf{J}_{s, z}=J_{s, z} \hat{\boldsymbol{y}}$ is induced in the piezospintronic material and injected into the NM. In turn this results in a spin accumulation $\mu_{z}(y)$ on the NM (translation symmetry in $z$ is assumed). Due to the spin-orbit coupling in the NM a transverse charge current $J_{q}$ will be generated along the $x$ direction, i.e., as an ISHE signal.

with strong spin-orbit coupling (SOC). As is well known, due to SOC, a charge current $\mathbf{J}_{q}$ flowing in the NM converts into a pure spin current (spin Hall effect), and vice versa (ISHE) [17]. Based on the above effect we expect to measure a charge Hall current as a result of the piezospin current induced at the interface. The process of injection of a spin current into a metal has been widely studied, for example, in Ref. [22]. We consider that a spin current is generated in the piezospintronic material with no loss of spin angular momentum in the bulk. Thus, the total spin current at the interface is $\mathbf{J}_{\sigma}^{s}(y=0)=\mathbf{J}_{s, \sigma}$. For simplicity, perfect transmission of spin current through the interface is assumed. Under this assumption we calculate analytically the charge current generated in the normal metal in terms of the spin current emitted from the piezospintronic material.

To analyze the connection between the spin and the charge currents in the metallic material we solve the spin diffusion equation for the spin accumulation [23] $\boldsymbol{\mu}_{s}(\mathrm{x})$,

$$
\nabla^{2} \boldsymbol{\mu}_{s}=\frac{\boldsymbol{\mu}_{s}}{\ell_{s}^{2}}
$$

where $\ell_{s}$ stands for the characteristic spin diffusion length of the NM. The boundary conditions for Eq. (6) enforce continuity for the spin current, which reads

$$
\begin{gathered}
\left.\partial_{y} \boldsymbol{\mu}_{s}\right|_{y=0}=-\frac{G_{0}}{\sigma} \mathbf{J}_{s, z}^{\mathrm{net}}, \\
\left.\partial_{y} \boldsymbol{\mu}_{s}\right|_{y=d}=0,
\end{gathered}
$$

where $G_{0}=2 e^{2} / h$ is the quantum of conductance, and $\sigma$ and $d$ are the conductivity and the thickness of the NM. $\mathbf{J}_{s, z}^{\text {net }}$ is the net spin current flowing through into the NM. The net spin current is the sum of the injected piezospin current, as given in Eq. (1), and a backflow spin current $\mathbf{J}_{s}^{\text {back }}$ in the opposite direction due to the induced spin accumulation on the NM side of the interface. In the calculation of the spin Hall current we disregard spin transfer torques generated by the 
spin current in the normal metal acting on the antiferromagnet. Moreover, without loss of generality the piezospin current is considered to flow in the $y$ direction and polarized along the $z$ axis. Additionally, in the bulk of the NM spin and charge currents are related through the relations

$$
\begin{aligned}
\mathbf{J}_{q} & =\frac{\sigma}{e} \nabla \mu-\frac{\sigma^{\prime}}{2 e} \nabla \times \boldsymbol{\mu}_{s}, \\
\frac{2 e}{\hbar} \mathbf{J}_{s, z} & =-\frac{\sigma}{2 e} \partial_{y} \boldsymbol{\mu}_{s}-\frac{\sigma^{\prime}}{e} \mathbf{z} \times \nabla \mu,
\end{aligned}
$$

with $\mu$ the electronic chemical potential and $\sigma^{\prime}$ the spin Hall conductivity in the NM [24]. Solving Eqs. (6)-(10) leads to an induced Hall charge current density along the $x$ direction

$$
\left\langle J_{q}^{x}\right\rangle=\Gamma \sum_{k l} \lambda_{z ; y k l} \frac{\mathrm{du}_{k l}}{\mathrm{~d} t},
$$

where $\Gamma=\frac{2 e}{\hbar} \frac{\ell_{s}}{d} \tan \theta_{H} \tanh \left(\frac{d}{2 \ell_{s}}\right)$, the Hall angle is $\theta_{H}=$ $\arctan \left[\sigma^{\prime} / \sigma\right]$, and $\langle\ldots\rangle$ denotes the thickness average. This effect can be measured by making use of materials with a huge potential for spintronic devices [26], for example, the transition-metal chalcogenophosphates $M \mathrm{P} X_{3}(M=\mathrm{V}$, $\mathrm{Mn} ; X=\mathrm{S}, \mathrm{Se}, \mathrm{Te})$ and $\mathrm{NiPSe}_{3}$. These materials are twodimensional semiconductors in which the transition-metal atoms of the compound are organized in a honeycomb lattice. Recent theoretical studies [27,28] have shown that these materials might exhibit a Néel order in the ground state which is not affected under strain. Nevertheless, this setup also works with materials without $\mathcal{T} \mathcal{I}$ symmetry. In this case there will be an additional piezoelectric response, but the charge current generated in that process will generate a transversal signal in the metal which will not affect the Hall signal.

It is worth commenting that a reciprocal effect is also expected. From Onsager's relations [29,30] a stress is expected in response to a spin current injected into the system,

$$
\mathbf{s}_{i j}=\sum_{l m} \tilde{\lambda}_{l ; m i j} \mathbf{J}_{l ; m}^{s},
$$

where $\mathbf{s}$ stands for the stress tensor [16] appearing in response to the spin current $\mathbf{J}^{s}$. This converse piezospintronic effect might lead to novel mechanisms for detecting pure spin currents. In fact, this effect might be useful in the mechanical resonance of the piezospintronic material. One idea is to consider an AF-ferromagnet interface with the ferromagnet under a ferromagnetic resonance. By spin pumping a spin current will be injected into the piezospintronic. With the proper excitation frequency, due to the reciprocal piezospintronic effect [see Eq. (11)], the piezospintronic material might get into a mechanical resonant state.

\section{DISCUSSION AND CONCLUSIONS}

In this paper we have discussed the possibility of generating and detecting pure spin currents via the piezospintronic effect in honeycomb antiferromagnets. We discuss the principal characteristics of this effect and the symmetry properties that lead to a pure spin current in response to strain. We characterize the piezospintronic response of a honeycomb antiferromagnetic layer, which fulfills the symmetry conditions to develop a pure piezospintronic response, and calculate its piezospintronic tensor. In the long-wavelength approximation we show that the relevant coefficients of the piezospintronic tensor are proportional to a Chern number. Finally, we propose an experimental setup to measure the spin current generated in this way by converting it into an electric current through the ISHE. This work extends the grounds for spin-mechanics [31] systems because it provides a direct coupling between spin current and strain.

\section{ACKNOWLEDGMENTS}

It is a pleasure to thank J. Fernández-Rossier for fruitful conversations. A.S.N. and C.U. appreciate the funding from Grant Fondecyt Regular 1150072. A.S.N. also acknowledges support from Financiamiento Basal para Centros Científicos y Tecnológicos de Excelencia (Chile), under Project No. FB 0807. R.E.T. is grateful for the funding from grants Fondecyt Postdoctorado 3150372 and Fondecyt Regular 1161403. R.A.D. is a member of the D-ITP consortium, a program of the Netherlands Organisation for Scientific Research (NWO) that is funded by the Dutch Ministry of Education, Culture and Science (OCW). This work was funded in part by the Stichting voor Fundamenteel Onderzoek der Materie (FOM).

\section{APPENDIX: CALCULATION OF THE BERRY PHASE}

To calculate the Berry phase we use the coherent states

$$
|\mathbf{n}\rangle=\left(\begin{array}{c}
e^{i \varphi} \cos \theta / 2 \\
\sin \theta / 2
\end{array}\right)
$$

along the direction of the vector

$$
\mathbf{n}=\left(\sum_{i} \mathrm{t}_{i} \cos \left(\boldsymbol{k} \cdot \vec{\delta}_{i}\right), \sum_{i} \mathrm{t}_{i} \sin \left(\boldsymbol{k} \cdot \vec{\delta}_{i}\right), \Delta\right),
$$

where $\theta$ and $\varphi$ are the polar and azimuthal angles, respectively, in spherical coordinates. In this representation Eq. (3) can be written as

$$
\mathbf{A}_{z, j}^{\mathrm{t}}=\int_{\mathrm{BZ}} \frac{\mathrm{d}^{2} k}{(2 \pi)^{2}} \operatorname{Im}\left\langle\nabla_{Q} \mathbf{n}|\times| \nabla_{Q} \mathbf{n}\right\rangle,
$$

where $Q=\mathrm{t}, k_{j}$. Hence, we can calculate the relevant contribution

$$
\mathbf{A}_{z, y}^{t_{3}}=\int_{\mathrm{BZ}} \frac{\mathrm{d}^{2} k}{(2 \pi)^{2}} \frac{\sin \theta}{4}\left(\frac{\partial \theta}{\partial \mathrm{t}_{3}} \frac{\partial \varphi}{\partial k_{y}}-\frac{\partial \theta}{\partial k_{y}} \frac{\partial \varphi}{\partial \mathrm{t}_{3}}\right) .
$$

The numerical solution of this integral is shown by the blue line in Fig. 3.

In the long-wavelength limit we perform an expansion of the eigenstates in $k$ around the Dirac point $K_{+}=\left(4 \pi / 3 \sqrt{3} a_{0}, 0\right)$. Around this point Eq. (A1) becomes

$$
\mathbf{n}_{K_{+}}=\left(-\frac{3}{2} a_{0} \mathrm{t} k_{x},-\frac{3}{2} a_{0} \mathrm{t} k_{y}, \Delta\right),
$$

which, following Eq. (A2), leads us to the expression

$$
\mathbf{A}_{z, y}^{\mathrm{t}_{3}}=-2 \int \frac{\mathrm{d}^{2} k}{(2 \pi)^{2}} \frac{3 a_{0} \mathrm{t}_{3} \Delta}{\left(9 a_{0}^{2} \mathrm{t}_{3}^{2}\left(k_{x}^{2}+k_{y}^{2}\right)+4 \Delta^{2}\right)^{3 / 2}} .
$$

The result of this integral is exactly Eq. (5) and is shown by the red line in Fig. 3. 
[1] J. Sinova and I. Žutić, Nat. Mater. 11, 368 (2012).

[2] R. Duine, Nat. Mater. 10, 344 (2011).

[3] S. A. Wolf, D. D. Awschalom, R. A. Buhrman, J. M. Daughton, S. Von Molnar, M. L. Roukes, and D. M. Treger, Science 294, 1488 (2001).

[4] A. S. Núñez, R. A. Duine, P. Haney, and A. H. MacDonald, Phys. Rev. B 73, 214426 (2006); A. H. MacDonald and M. Tsoi, Philos. Trans. A. Math. Phys. Eng. Sci. 369, 3098 (2011).

[5] T. Jungwirth, X. Marti, P. Wadley, and J. Wunderlich, Nature Nanotechnol. 11, 231 (2016); V. Baltz, A. Manchon, M. Tsoi, T. Moriyama, T. Ono, and Y. Tserkovnyak, arXiv:1606.04284v2 [cond-mat].

[6] A. V. Kimel, A. Kirilyuk, P. A. Usachev, R. V. Pisarev, A. M. Balbashov, and Th. Rasing, Nature (London) 435, 655 (2005); Nat. Phys. 5, 727 (2009); T. Satoh, S. J. Cho, R. Iida, T. Shimura, K. Kuroda, H. Ueda, Y. Ueda, B. A. Ivanov, F. Nori, and M. Fiebig, Phys. Rev. Lett. 105, 077402 (2010); S. Wienholdt, D. Hinzke, and U. Nowak, ibid. 108, 247207 (2012).

[7] E. M. Lifshitz and L. P. Pitaevskii, Statistical Physics. Course of Theoretical Physics, Vol. 9 (Pergamon Press, Oxford, UK, 1980).

[8] P. Tang, Q. Zhou, G. Xu, and S.-C. Zhang, Nat. Phys. 12, 1100 (2016); L. Šmejkal, T. Jungwirth, and J. Sinova, Phys. Status Solidi RRL 11, 1700044 (2017).

[9] R. S. K. Mong, A. M. Essin, and J. E. Moore, Phys. Rev. B 81, 245209 (2010).

[10] R. Cheng, J. Xiao, Q. Niu, and A. Brataas, Phys. Rev. Lett. 113, 057601 (2014).

[11] A. S. Nunez, Solid State Commun. 198, 18 (2014).

[12] W. G. Cady, Piezoelectricity: An Introduction To The Theory And Application Of Electromechanical Phenomena In Crystals (McGraw-Hill, New York, 1946); L. D. Landau, J. S. Bell, M. J. Kearsley, L. P. Pitaevskii, E. M. Lifshitz, and J. B. Sykes, Electrodynamics of Continuous Media, Vol. 8 (Elsevier, Amsterdam, 2013).

[13] I. E. Dzialoshinskii, J. Exptl. Theoret. Phys. (U.S.S.R.) 33, 807 (1957).

[14] J. Shi, P. Zhang, D. Xiao, and Q. Niu, Phys. Rev. Lett. 96, 076604 (2006).
[15] J. F. Nye, Physical Properties of Crystals: Their Representation by Tensors and Matrices (Oxford University Press, New York, 1985).

[16] L. D. Landau and E. M. Lifshitz, Theory of Elasticity, 3rd ed. Course of Theoretical Physics, Vol. 7 (Butterworth-Heinemann, Oxford, UK, 1986), p. 3.

[17] E. Saitoh, M. Ueda, H. Miyajima, and G. Tatara, Appl. Phys. Lett. 88, 182509 (2006); T. Kimura, Y. Otani, T. Sato, S. Takahashi, and S. Maekawa, Phys. Rev. Lett. 98, 156601 (2007).

[18] M. Droth, G. Burkard, and V. M. Pereira, Phys. Rev. B 94, 075404 (2016).

[19] R. Resta and D. Vanderbilt, Phys. Ferroelectr.: A Mod. Perspect. 105, 31 (2007).

[20] R. D. King-Smith and D. Vanderbilt, Phys. Rev. B 47, 1651 (1993).

[21] D. J. Thouless, M. Kohmoto, M. P. Nightingale, and M. den Nijs, Phys. Rev. Lett. 49, 405 (1982).

[22] F. J. Jedema, A. T. Filip, and B. J. van Wees, Nature 410, 345 (2001); F. J. Jedema, M. S. Nijboer, A. T. Filip, and B. J. van Wees, Phys. Rev. B 67, 085319 (2003).

[23] V. Zayets, Phys. Rev. B 86, 174415 (2012).

[24] Y. Tserkovnyak and S. A. Bender, Phys. Rev. B 90, 014428 (2014).

[25] K. Uchida, S. Takahashi, K. Harii, J. Ieda, W. Koshibae, K. Ando, S. Maekawa, and E. Saitoh, Nature 455, 778 (2008).

[26] X. Li and W. Xiaojun, Wiley Interdisciplinary Reviews: Computational Molecular Science 6.4 (Wiley, New York, 2016), pp. 441-455.

[27] B. L. Chittari, Y. Park, D. Lee, M. Han, A. H. MacDonald, E. Hwang, and J. Jung, Phys. Rev. B 94, 184428 (2016).

[28] J. U. Lee, S. Lee, J. H. Ryoo, S. Kang, T. Y. Kim, P. Kim, C. H. Park, J. G. Park, and H. Cheong, Nano Lett. 16, 7433 (2016).

[29] L. Onsager, Phys. Rev. 37, 405 (1931).

[30] L. D. Landau and E. M. Lifshitz, Statistical Physics, 3rd ed., Part I. Course of Theoretical Physics, Vol. 5 (ButterworthHeinemann, Oxford, UK, 1980).

[31] H. Keshtgar, M. Zareyan, and G. E. W. Bauer, Solid State Commun. 198, 30 (2014); P. Chowdhury, A. Jander, and P. Dhagat, arXiv:1702.06038 [cond-mat.mes-hall]. 